\title{
RE(DE)FINING PROJECT DELIVERY WITH LIFE CYCLE BIM
}

\begin{abstract}
Adam Rendek $^{1}$
Abstract: This paper explores a shift in the Architecture, Engineering, Construction (AEC), and Capital Facilities Industries in which all stakeholders of the building life cycle unite around forming common goals. We are witnessing changes in these industries due to recent economic, cultural, and technical development, and these changes provide an opportunity to re-think and re-define project delivery more efficiently.

We will review influencing stakeholder groups and their relationships or the lack of. The paper will also offer specific examples where BIM (Building Information Modeling) can directly support greater collaboration and promote the complete building information life cycle. The assumptions are based on the experiences of working on large capital projects in California, although the references to literature from the past few years highlight international trends as well.

Assumptions and conclusions in this paper are based on action research and interviews with stakeholders of large public organizations during the implementation of building owner side BIM programs.
\end{abstract}

Keywords: Project delivery, collaboration, BIM, communication, complete building information life cycle.

\section{INTRODUCTION}

\subsection{Changes in project delivery}

Changes in popular project delivery methods have been promoting collaboration for decades, however significant misalignments continue to exist between stakeholder groups, resulting in inefficiencies. In parallel, today's technology can effectively support communication and coordination, but technology alone cannot solve these issues unless we also address stakeholder organization.

In this paper, we will look at current project delivery types, the connection between stakeholders and trends in technology adoption, which will provide a better understanding of project organization. Building on that, we will use practical examples where BIM can directly support collaboration.

There are many types of project delivery. Let us explore three in use in the USA to illustrate the evolution of project delivery from the perspective of collaboration.

- Design-Bid-Build (D/B/B) project delivery has separate phases with limited overlap and collaboration between them.

- Design-Build $(D / B)$ has been established to allow early constructability input. Designers and construction professionals work together in this project delivery to achieve a coordinated result.

BIM/ Engagement Manager, DPR Construction, San Francisco, CA, USA, adamre@dpr.com 
- Integrated Project Delivery (IPD) builds further on collaboration with solutions, for example co-locating designers, engineers, and builders in "big rooms".

Concurrent collaboration is more prominent as we move from $D / B / B$ to $I P D$. The incentive to collaborate is primarily financial in nature, which can cause the separation of stakeholder interests when problems arise. It needs to be acknowledged that designing and building facilities is a business activity and therefore financial outcome is an important consideration.

The changes in project delivery were in fact driven by economic considerations; to achieve better, less expensive results, faster. This was especially true after the 2008 economic crisis.

\subsection{Advancements in project team collaboration}

Lean practitioners reported that the alignment between project participants are as equally important as efficient scheduling (McGraw Hill Construction 2013). The General Services Administration (GSA), which is the largest building owner in the USA has adopted the concept of Partnering to support communication and to align expectations between stakeholders (GSA n.d.).

Similarly, the International Partnering Institute (IPI) highlights that: "Through structured Partnering, fragmented teams coalesce and unify around a shared objective: successful project delivery" (IPI 2016).

While the concept of Partnering is not new, the recognition of the need to connect stakeholder groups and organize them around a common objective is an important one. A growing number of building owners or organizations working primarily with building owners initiate Partnering as the foundation of project team organization. This is also significant because the active participation of building owners is needed to create a complete building information life cycle.

The question is, if improvements in collaboration have been part of the project delivery evolution, why are there still significant disconnects between stakeholders? Our hypothesis is that detailed collaboration has been mostly led by the design and construction community in the past decades, with limited oversight from the building owners.

This has resulted in a focus on active collaboration during design and construction with a clear goal of successful handover. Handover generally being defined as the end of project life, when financial transactions were completed, and building owners had started a new phase of operations.

The attempt to extend the project life cycle to include operations received limited understanding and incentives. We will provide reasons for this in the next part of the paper while reviewing the connection between stakeholders.

There have been recent advances in organizing and collecting information during design and construction that are directly useful in operations, but this effort has mainly focused on facilities maintenance.

As we will discuss it later, a holistic look at the stakeholder subgroups on the building owner side would be necessary to connect design and construction with operations. These subgroups include occupants, capital investment representatives, and operations stakeholders. 


\section{STAKEHOLDERS}

\subsection{Perceived issues}

The boundaries between disciplines and divisions between project phases exist for good reasons. For example, the Design-Bid-Build delivery method is not simply inefficient, it was established to serve specific purposes (e.g. fair, public competition). "Lean thinking views the entire project in production system terms, that is, as if the project were one large operation" (Howell and Ballard 1998). However, adopting new technologies or collaboration methods that cross these boundaries and divisions can present difficulties without considering the original reasons behind the boundaries and divisions.

One of the cornerstones of partnering is commitment by all participants. Committing to something is only possible if the subject is well-understood. Lack of willing collaboration between stakeholders can stem from concerns about professional liability or code issues which need to be observed. Vaguely defined requirements from owners can also contribute to sub-optimal results. Team-work can break down when problems arise and the initial commitment was not based on a known effort.

For these and other reasons, one stakeholder group can refrain from committing time and effort to the process.

\subsection{Stakeholder groups}

There are many disciplines and stakeholder groups involved in developing and executing a building project. The different disciplines often represent different work cultures, and can associate different meanings to concepts that appear to be the same on the surface.

For example, we explored the communication between architects and energy modelers during the development of high-efficiency buildings. "As they leave the meeting table, architects and engineers have a different expectation about what the other side is going to deliver to the next meeting or how much effort the identified next exercise will take" (Grinberg and Rendek 2013).

To further complicate matters, stakeholders often have subgroups with their own cultures and differences. They are tasked to represent their own subject matters and coordinate with other disciplines and stakeholders. Table 1 lists the different focus areas of the various participants of a hypothetical Design-Build project to illustrate the diversity of special interests during any building project. 
Table 1: Diversity of stakeholder interests.

\begin{tabular}{ccc}
\hline Stakeholder & Subgroup & Examples of Special Interest \\
\hline Building Owner & Capital Planning & Best value for investment \\
& Occupants/ Users & Occupant comfort, usability of facility \\
& Capital Projects & Schedule and cost of building \\
& Facilities Maintenance & Feasibility and cost of maintenance \\
& Operations & Asset management, operations \\
Permitting Authority & & Code compliance, public safety \\
General Contractor & & Schedule and cost of building, \\
Engineer/ Subcontractor & Mechanical, Electrical, & Subsystem performance, constructability, \\
& etc. & code compliance \\
Architect & & Design intent, code compliance \\
& Design & Space and building design \\
& Planning & Program fitting, adjacency \\
\hline
\end{tabular}

Building owners can play an important role in facilitating team alignment when they dedicate resources and personnel to developing a deeper knowledge of the areas that affect multiple stakeholder groups or multiple building life cycle phases (Dodge Data \& Analytics 2016).

Let us take the example of facilitating collaboration around building information that has a direct effect on operations or facilities maintenance. This requires understanding of where the information originates from, in what format it needs to be developed, and what supports the handover between project phases and stakeholders.

In turn, building owners can also provide clear reasons for why the information is needed in a certain way which promotes a willingness to collaborate around it. Even if the stakeholder group leaders commit to coordination with an understanding of the details, they need to continue to actively support their practitioners who execute these tasks daily.

The rise of the Design-Build and the Integrated Project Delivery methods has drawn attention to the coordination between designers, construction professionals, and engineers. Here, we will focus on the involvement of the permitting authority and the building owner.

The permitting authority can be the single most influential stakeholder in a building project. For example, the schedules of large healthcare projects in California often revolve around the permitting process of the Office of Statewide Health Planning and Development (OSHPD) due to the lengthy nature of that process.

In this case, an understanding of the local authority's requirements needs to be shared between architects, engineers, subcontractors, general contractors, and the owner capital project managers. The whole project team needs to have aligned expectations and the project schedule needs to include added time for effective execution (Santorella 2011).

The Singapore Building \& Construction Authority's BIM Initiative was one of the first permitting authority-led developments to use BIM for a more efficient review and approval process. Although the program has been generally successful and still running after many 
years, the disconnect between stakeholders happens to be on the design and construction side. The designers and construction professionals in Singapore are adopting BIM slower than was expected. Therefore, they cannot feed the authority's system at the rate and efficiency that was originally envisioned.

The Taiwanese government is also considering BIM-based e-submission for building permits. In their case "more than half of the firms were willing to use BIM-based tools to streamline the building permit review process" (Juan et al. 2016).

The permitting process can create a bottleneck in the schedule of building projects and, for the same reason, it can provide an opportunity for time savings after careful alignment of all stakeholders and clear understanding of local requirements and culture. Time savings leading to early operations, in turn, can have significant positive financial results for the owner capital project managers. We will discuss practical BIM uses that can support the permitting authority's work and coordination with other stakeholders in the next part of this paper.

While organized data from design and construction to support facilities maintenance has documented benefits (McGraw Hill Construction 2014), the results are difficult to illustrate. Large building owners typically have separate stakeholder groups representing capital project investment and facilities maintenance. These internal groups are often funded separately and siloed in ways that slows the implementation of a complete building information life cycle. The capital project manager's focus is finishing the project on time and within budget.

While most of them consider long term maintenance to achieve best value for the investment, they are not directly incentivized to adopt BIM to support it, especially if it is at the cost of an extended schedule or inflated budget. The few organizations at the forefront of developing a complete building information life cycle recognize this issue and actively work on connecting their internal stakeholders (Dodge Data \& Analytics 2016b).

Occupants and users on the owner side can also benefit from better coordination and the adoption of technology that supports it. Taking the healthcare sector as an example once more, large projects may need hundreds of user meetings in early design phases. These meetings require not just time, but a significant amount of personnel and financial resources, making them ideal environments for improvement through better coordination.

\section{EFFECTIVE SUPPORT BY TECHNOLOGY}

Once collaboration and the alignment of expectations are addressed between stakeholders, it is possible to choose the right technology to support the right tasks. BIM is no exception from this and its use needs to be evaluated accordingly.

\subsection{Partnering with Virtual Design and Construction (VDC)}

As we explored above, the adoption of Partnering as a method of structured collaboration is increasing. Thoughtful commitment to collaboration and leaders supporting practitioners on the ground ensures better results in difficult circumstances. While financial performance is important, making the effort to understand different stakeholders' special interests will result in better execution, increase the willingness for collaboration, and can therefore positively impact financial results as well.

Virtual Design and Construction (VDC) addresses processes and organization in design and construction and it integrates BIM as key technology in a measurable way through multidisciplinary collaboration (Kunz and Fisher 2012). 
VDC has been developed at Stanford University's Center for Integrated Facility Engineering (CIFE) since 2001, and adopted by several design and construction firms with documented results (Kam et al. 2013).

Partnering and VDC are naturally aligned because of the complementing nature of their key components. Jointly, they provide a good reference point for building owners who want to adopt BIM for operations and plan to actively collaborate with their project teams.

\subsection{Practical examples of BIM use}

Stakeholders on the building owner side may not be familiar with the benefits of BIM or how it can support their work because primarily designers and construction professionals have used it in the past decades. For this reason, clearly defining project specific BIM uses and their potential impact can help to align expectations between building owners, occupants, and project team members. If this alignment happens early in the project, BIM can be a tool to support scope and requirement clarification through handover.

Visualization is an example of BIM application that can be tailored to support the needs of multiple project stakeholders. Whether the subject is 3D utility coordination, building security concerns, or the esthetic review of design, BIM based visualization can help to align the expectations of building owners and occupants, and design and construction professionals.

The following are a few additional practical examples where BIM can directly support collaboration and the appropriate alignment of needs and expectations.

\subsubsection{Accessing Building Information during Operations}

The building owners of large organizations with multiple buildings and campuses can benefit from standards addressing coordinate system alignment. BIM is designed to assist the design and documentation of individual buildings.

The location of buildings can be recorded in BIM, but due to limited need, it is not current standard practice. Building owners need to develop a process to achieve that, which in turn can be followed by various project teams creating virtual models.

Geospatial Information Systems (GIS) are often deployed by building campus owners. When GIS is connected to BIM, they provide a platform for accessing building information from multiple buildings at once without having to open individual BIM files.

\subsubsection{BIM supporting permitting}

Permitting authorities can benefit from viewing BIM as a database with graphic representation. Standardized attributes of wall types can be filtered to highlight fire rated walls and the related components. This can expedite the review of code compliance and fire life safety strategies.

Compliance with the American Disability Act (ADA) can also be facilitated in a similar fashion. Designers can benefit from using intelligent BIM elements that automatically display clearance issues without manual calculations.

Visualization based on BIM can generally support the permit review process, especially $3 \mathrm{D}$ views, which can lead to faster understanding of the designer intent.

\subsubsection{Connecting the virtual and the real}

QR codes and passive RFID chips are inexpensive today and can be easily attached to equipment and elements of the building. Every BIM element is coded with a unique identifier by default which can be linked to the QR codes or RFID chips of their physical 
equivalent during commissioning. This connection allows for automated equipment tracking or identification during operations and maintenance saving the time of manually searching for the element in drawings and documents.

\section{CONCLUSIONS}

\subsection{Balance and clarity}

The adoption of BIM has been unfolding in phases in the past decades. As the design and construction community has demonstrated its benefits, building owners and permitting authorities became interested in its use as well.

Five years ago, it was usual for building owners to request BIM without really understanding it. Today, we are approaching a tipping point when building owners and permitting authorities will ask for BIM and know how to use it as well.

This is signalled by several large building owners and permitting authorities developing specific requirements and establishing data standards where they don't currently exist. Complete building information life cycle is possible when building owners actively participate in team organization, provide detailed data standards and requirements, and effectively use the organized information through operations. This way, collaboration through Partnering can be balanced with choosing the right technologies for the right tasks. Permitting authorities can also support and benefit from this process which can affect a capital project's bottom line.

\subsection{Proposed next steps}

A pilot project designed around thoughtful collaboration, including all stakeholders and aimed at complete building information life cycle, could enhance our understanding of the challenges and benefits of the process, data development, and data use. While further research supporting facilities maintenance with standardized data is needed, we are proposing a focused effort to explore the benefits of using BIM by capital project managers, building users, and permitting authorities.

\section{REFERENCES}

Dodge Data \& Analytics (2016). Optimizing the Owner Organization, The Impact of Policies and Practices on Performance SmartMarket Brief, s.l.: Dodge Data \& Analytics. McGraw Hill Construction (2013). Lean Construction, Leveraging Collaboration and Advanced Practices to Increase Project Efficiency SmartMarket Report, s.l.: McGraw Hill Construction.

McGraw Hill Construction (2014). The Business Value of BIM for Owners SmartMarket Report, s.l.: McGraw Hill Construction. Grinberg, M. and Rendek, A. (2013). Architecture \& Energy in Practice: Implementing an Information Sharing Workflow. Chambery/ France, 13th Conference of International Building Performance Simulation Association.

GSA (n.d.) Partnering. [Online]

Available at: https://www.gsa.gov/portal/content/100822

[Accessed 27 March 2017].

Howell, G. and Ballard, G. (1998). Implementing Lean Construction: Understanding and Action. Guaruja, Brazil, The International Group for Lean Construction. 
IPI (2016). What is Construction Partnering? [Online]

Available at: https://partneringinstitute.org/what-is-collaborative-partnering/ [Accessed 27 March 2017].

Juan, Y.-K., Lai, W.-Y. and Shih, S.-G. (2016). Building information modeling acceptance and readiness assessment in Taiwanese architectural firms. Journal of Civil Engineering and Management, 2016(June), pp. 1-12.

Kam, C., Senaratna, D., Xiao, Y. and McKinney, B. (2013). The VDC Scorecard: Evaluation of AEC Projects and Industry Trends, s.l.: Stanford University CIFE.

Kunz, J. and Fisher, M. (2012). Virtual Design and Construction: Themes, Case Studies and Implementation Suggestions, s.l.: Stanford University CIFE.

Santorella, G. (2011). Lean Culture for the Construction Industry: Building Responsible and Committed Project Teams. New York: Taylor and Francis Group, LLC. 